\title{
A Blind Watermarking Algorithm with Semantic Meaningful Watermarks
}

\author{
Chun-Hsiang Huang, Ja-Ling Wu, Senior Member, IEEE, and Ding-Yun Chen \\ Communication \& Multimedia Laboratory \\ Department of Computer Science and Information Engineering \\ National Taiwan University, Taipei, Taiwan \\ $\{$ bh, wjl,dynamic\}@cmlab.csie.ntu.edu.tw
}

\begin{abstract}
Watermarking techniques that need no original information during watermark detection, the so-called blind (public) watermarking, are more desirable than others for practical usage and convenience in watermark extraction. In this paper, we convert a non-blind (private) image watermarking algorithm into a blind one by carefully removing and changing some function units of the original approach. As compared to the original algorithm, these modifications result in little quality degradation but do extend the practical usage of it. As an example, we applied the revised watermarking algorithm to a panorama picture construction tool, the VideoVR system, in which only blind watermarking algorithms can be used. The experimental results show that almost no perceptible distortions are found in the embedded pictures, and the retrieved watermarks can clearly indicate the ownership of the system constructor.
\end{abstract}

\section{The DCT-based Image Watermarking System}

Watermarks of the original non-blind algorithm [1] are semantically meaningful patterns (binary images) that can be directly recognized by naked eyes. As shown in Fig. 1, watermarks are embedded in the DCT domain of the original image. To obtain better image quality, both the original and watermark images are adequately partitioned

0-7803-6514-3/00/\$10.00@2000 IEEE

1827 into blocks. After sorting the original image blocks based on their variance value in the spatial domain, watermark blocks having more embedding positions are mapped to the image blocks with higher variances. The mapping mechanism is helpful in getting better imperceptibility, but it also result in the indispensability of original images during watermark extraction. Since the variance value of each block will be changed after watermark embedding, the sorted order of each block according to their variance value will be different from the one before embedding. Thus, the only way to find out the mapping information is to recalculate it from the original images. Furthermore, in [1], watermarks are embedded by reversing the inequality relationship (polarity) between the embedded DCT coefficients and the DC values (both of them are scaled and JPEG-quantized). To be more specific, if a watermark bit is 1 , the polarity in the corresponding embedding position is reversed; if a watermark bit is 0 , the polarity is left unchanged. To extract a watermark, both the original image and the embedded image are transformed into the DCT domain, and the DCT coefficients in corresponding positions are XORed to find out all polarity disagreements. Though this watermarking algorithm can achieve good imperceptibility and robustness, the need of original images during watermark extraction limits its practical usage. In section II, we will introduce a real case in which only blind watermarking techniques can be applied. 


\section{An Example: Watermarking for the VideoVR System}

The VideoVR system [2] is a panorama-picture construction tool, developed in the Communication and Multimedia Laboratory, National Taiwan University. To protect the Intellectual Property Rights (IPR) of the system developers, we would like to include a watermark-embedding module in the VideoVR system, such that an invisible watermark will be added in each output panorama picture to indicate the IPR claims. However, because only panorama pictures finalized by system users are published to the public, original images without embedding watermarks are not available. Therefore, only blind watermarking techniques can be used to protect the copyrights of system developers. To use the original DCT-based watermarking algorithm for the VideoVR system, the watermarking algorithm should be modified into a blind one.

\section{Modifications}

First, we simply remove all function units related to the aforecited variance mapping mechanism. Though image quality after embedding is expected to degrade severely, the experimental results show that the effect can be relieved by carefully adjusting the embedding parameters, such as the embedding energy within each block. Besides the removal, we adopt a watermarking labeling technique similar to the one in [3] to replace the original polarity reversing process: now the polarities of DCT coefficients are labeled according to the watermark sequences. That is, if a watermark is 1 , the polarity is set to positive, regardless of the original inequality relationship. If a watermark is 0 , the polarity is set to negative. The original image is not needed any more, because the watermark sequence can be easily read out from the embedded image alone. Figure 2. shows the block diagrams of the newly proposed blind watermarking system.

\section{Experimental Results and Conclusions}

From the experimental results, embedded watermarks are robust to common image operations for panorama pictures; moreover, output images have little perceptible defects after embedding. Fig. 3 shows the snapshots of the VideoVR output images before (Fig. 3a) and after (Fig. 3b) watermarking, and the extracted watermark images (Fig. 4)

In summary, we have successfully converted a non-blind watermarking algorithm into a blind one, and use it as a feasible copyright protection mechanism for commercial products. A more powerful version of VideoVR watermarking system that can resist shift-attacks is introduced in [4]. In addition to VideoVR, we believe that the simple algorithm could be easily applied to video data and thus greatly expand the scope of the original algorithm's applications.

References

[1] C. T. Hsu and J. L. Wu, "Hidden digital watermarks in images, " IEEE Trans. on Image Processing, vol 8., No. 1, Jan. 1999.

[2] D. Y. Chen, M. Ouhyoung, "VideoVR: A real-time system for automatically constructing panoramic images from video clips", IFIP 1998 Workshop on Modeling and Motion Capture Techniques for Virtual Environments (C'APTECH98), Geneva, Switzerland, Nov. 1998.

[3] G. C. Langelaar, R.L. Lagendijk, and J. Biemond, "Real-time labeling of MPEG-2 compressed video", Journal of Visual Communication and Image Representation, Dec. 1998.

[4] D. Y. Chen, C. H. Huang, J. L. Wu, M. Ouhyoung, "A shift-resisting blind watermark system for panoramic images", Proc. IEEE International Conference on Consumer Electronics (ICCE 2000), June, LA. , pp.8-9, Jun. 2000. 


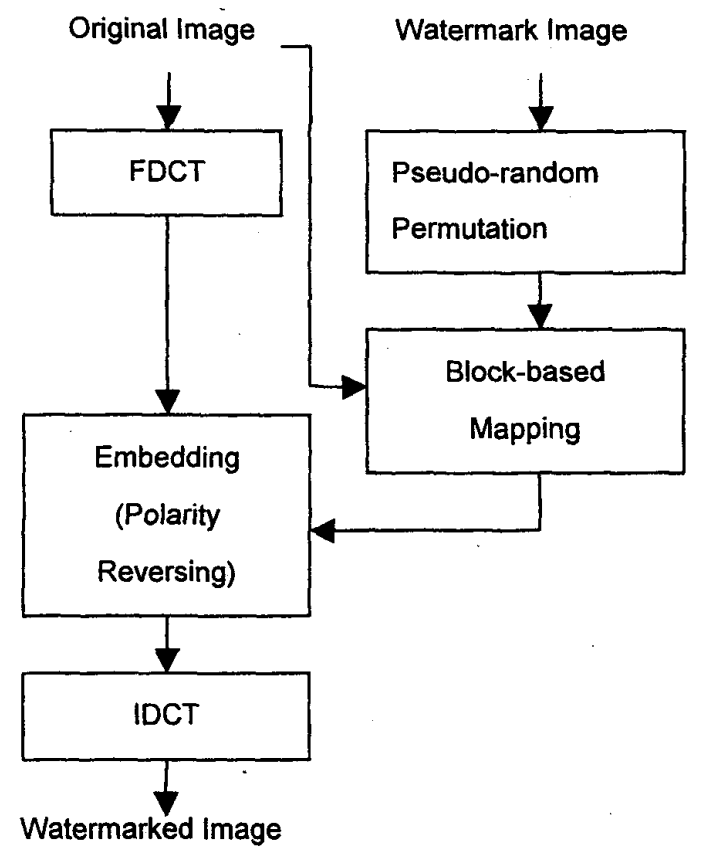

Figure 1(a): Watermark embedding processes of the DCT-based watermarking system

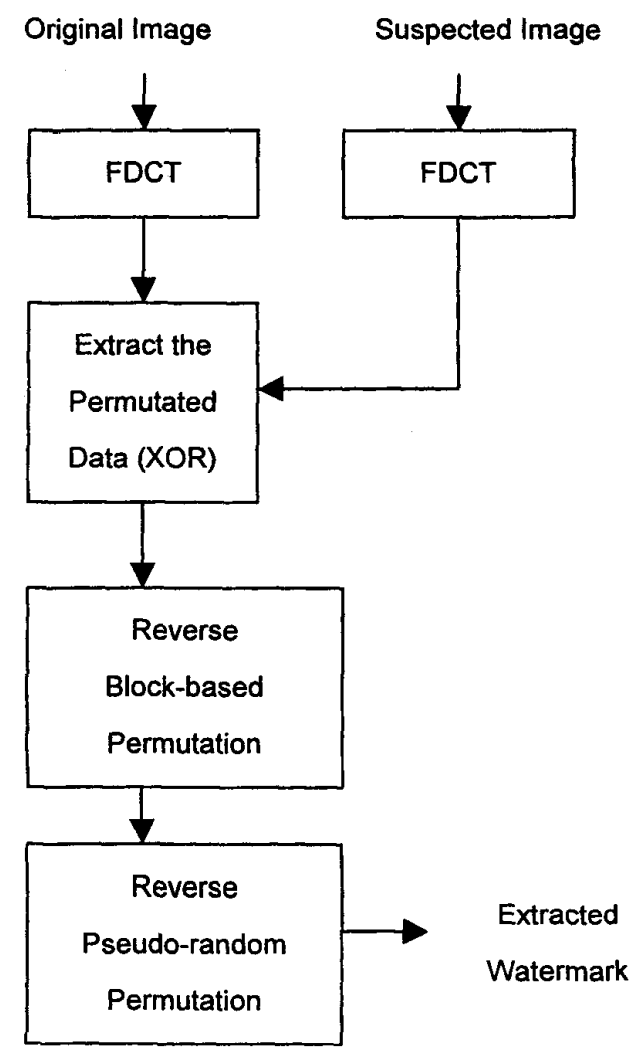

Figure 1(b):Watermark Extraction Processes of the DCT-based Watermarking System

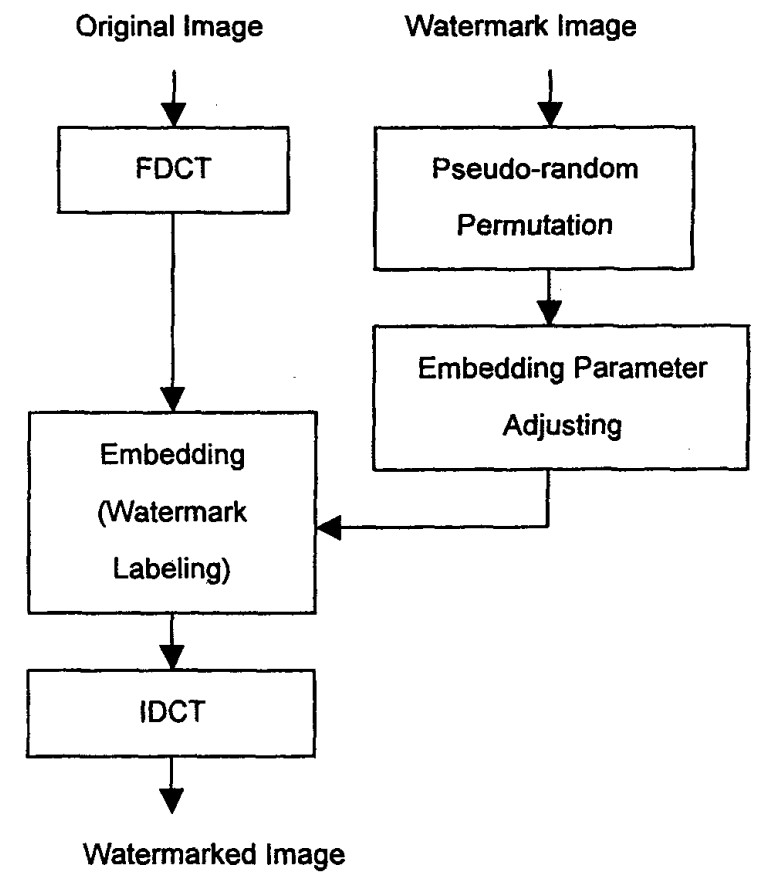

Figure 2(a): Watermark embedding processes of the blind watermarking system

Suspected Image

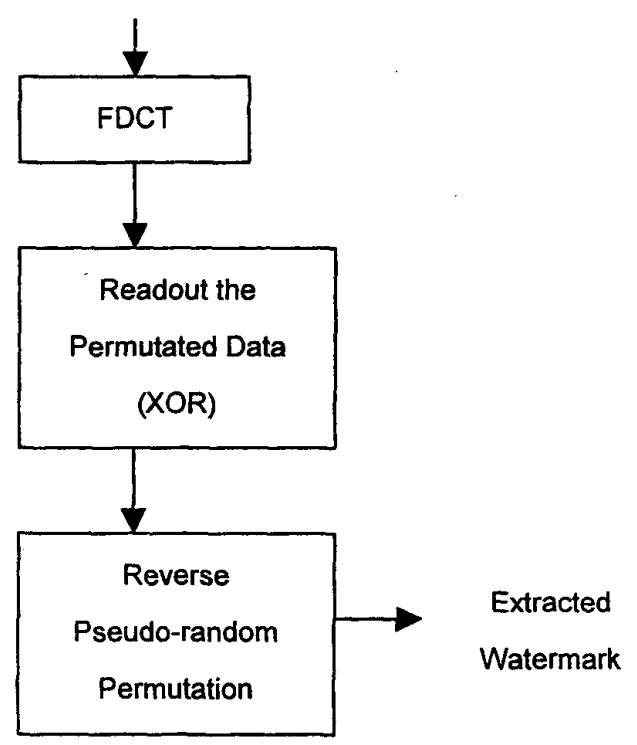

Figure 2(b): Watermark extraction processes of the blind watermarking system 

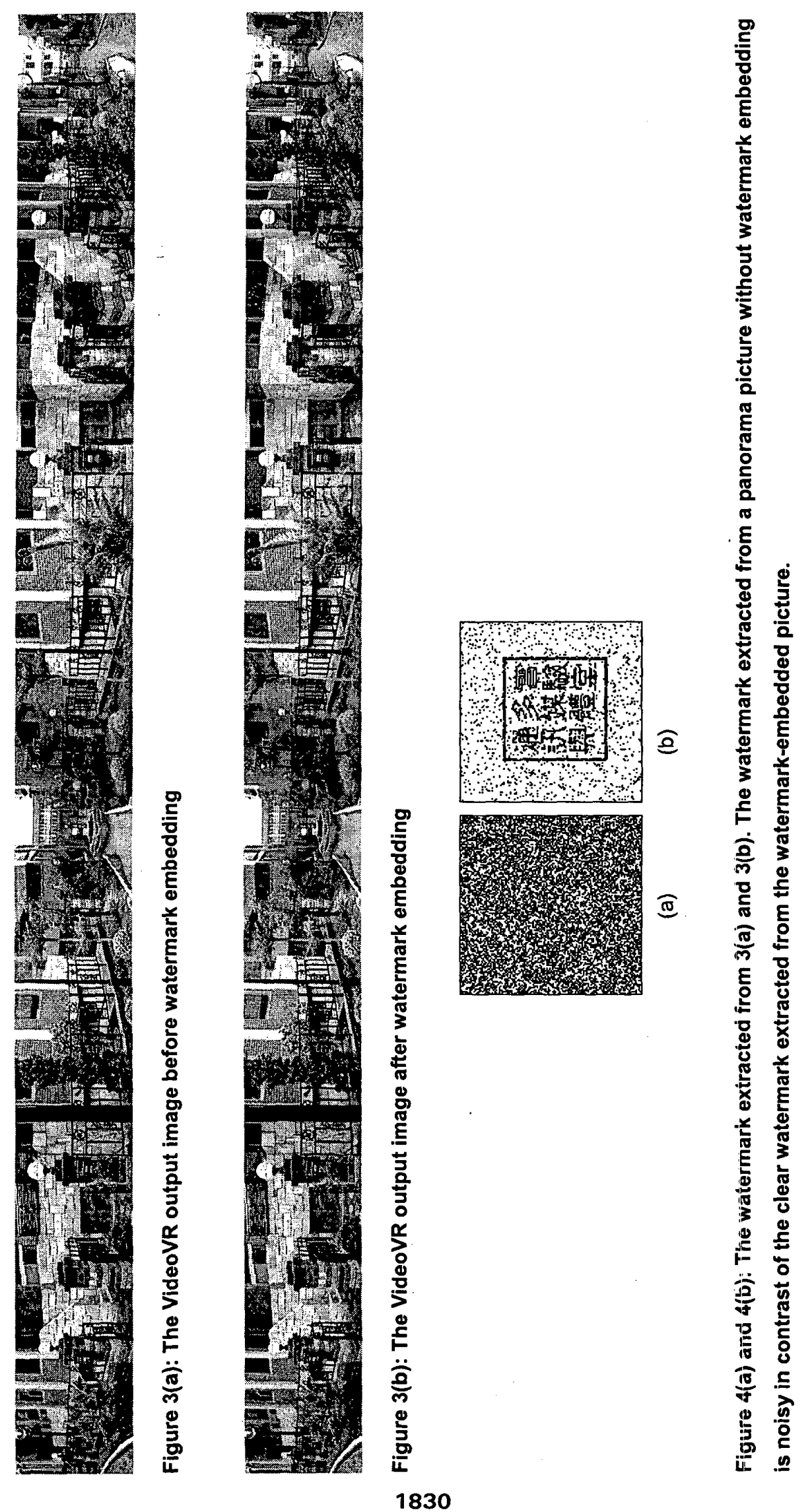